\title{
PERCEPCIÓN DE INVESTIGADORES NACIONALES EN MÉXICO DEL ÁREA DE HUMANIDADES SOBRE PRINCIPIOS ÉTICOS Y CONDUCTAS ÉTICAS INACEPTABLES
}

Esthela Carrillo Cisneros, ${ }^{1}$ Sonia Guadalupe Zermeño Flores ${ }^{2}$ y Carmen Patricia Jiménez Terrazas ${ }^{3}$

\begin{abstract}
Resumen: El marco de referencia de ética en la investigación en humanidades y ciencias sociales es relativamente reciente y aún hay países que no incluyen en su práctica de investigación pautas éticas para esta disciplina. Debido a esto, son escasos los estudios donde se conozcan las percepciones de investigadores de estas disciplinas relacionados a las situaciones éticas sobre su práctica de investigación ni tampoco sobre lo que para ellos representa, por lo que se aborda el tema con investigadores del área IV que pertenecen al Sistema Nacional de Investigadores (SNI) en México, desde un enfoque cualitativo, mediante entrevistas personales semi-estructuradas. Con una muestra de 12 participantes, los resultados revelan su experiencia con principios éticos y enumeran conductas éticas inaceptables en las distintas etapas de investigación, arbitraje y publicación. Sobre la ética de la investigación en México, unos hablan de burocracia y falta de financiamiento y otros, sobre esfuerzos profundos por parte de CONACyT, así como la necesidad de crear códigos éticos del área.
\end{abstract}

Palabras clave: investigación, ética, humanidades

\section{PERCEPTION OF NATIONAL RESEARCHERS IN MEXICO FROM THE HUMANITIES AREA ON ETHICAL PRINCIPLES AND UNACCEPTABLE ETHICAL BEHAVIOR}

\begin{abstract}
The framework of ethics in research in humanities and social sciences is relatively recent and there are still countries that do not include in their research practice ethical guidelines for these disciplines. Due to this, there are few studies where the researchers' perceptions of these disciplines related to the ethical situations about their research practice are known, nor what it represents for them, so the approaches of the topic with researchers belonging to area IV to the National Researchers System in Mexico, from a qualitative approach through semistructured personal interviews. With a sample of 12 participants, the results reveal their experiences with ethical principles, they list unacceptable ethical behavior in the different stages of research, arbitration and publication. On the ethics of research in Mexico, some speak of bureaucracy and lack of funding and others, of deep efforts by Conacyt, as well as the need to create ethical codes of the area.
\end{abstract}

Keywords: research, ethic, humanities.

\footnotetext{
${ }^{1}$ Doctora en Ciencias Administrativas; profesora-investigadora en la Universidad Estatal de Sonora.

2 Doctora en Ciencias Administrativas; profesora-investigadora en la Universidad Estatal de Sonora.

${ }^{3}$ Doctora en Ciencias de la Administración; profesora-investigadora en la Universidad Autónoma de Ciudad Juárez; S.N.I. I; pjimenez@uacj.mx.
} 


\section{INTRODUCCIÓN}

Los principios y normas de la ética en la investigación se enfocaron desde mediados de los 50`s del siglo XX en áreas médicas y, posteriormente, en otras áreas de la ciencia. La ética en la investigación en las humanidades comenzó a regularse en algunos países hasta la década de los 70’s, cuando — por ejemplo - en 1978, en Canadá, se instaló el Consejo de Investigación de Ciencias Sociales y Humanidades; y en 1974, en Estados Unidos, se creó la Comisión Nacional de Protección para las personas en temas de investigación biomédica y de comportamiento.

En países europeos (como Inglaterra), también fue en esa década cuando su Consejo de Investigación Médica mencionó, por primera ocasión —en un documento, en 1975- la investigación en humanidades y ciencias sociales (Van Den Hoonaard, 2011).

En ese sentido, algunas de las quejas por parte de investigadores en humanidades, se refieren a que las guías de ética en la investigación donde se mencionaba explícitamente a las humanidades y ciencias sociales, tienen su origen en la investigación médica y clínica; por lo que los comités de ética los forzaban a enmarcar sus protocolos de investigación y procedimientos de revisión ética, bajo modelos clínicos o cuantitativos para satisfacer estos requerimientos, sin considerar las características propias de la investigación en humanidades y ciencias sociales (Cribb, 2004; Zito Guerriero y Magalháes Bosi, 2015; Wynn, Israel, Thomson, White y Carey-White, 2014).

De acuerdo a los criterios específicos del marco legal del sistema nacional de investigadores en México, el área cuatro de humanidades y ciencias de la conducta, incluye las disciplinas de: Antropología, Antropología Física, Arqueología, Arquitectura, Artes y Letras, Bibliotecología, Diseño, Filosofía, Historia, Historia del Arte, Lingüística, Matemáticas Educativas y Enseñanza de las Ciencias, Pedagogía y Psicología (Área IV SIN, 2016).

En los últimos años, estas objeciones han tenido una mayor repercusión, de tal suerte que algunos países — como Estados Unidos, Canadá, Australia, Finlandia, Noruega, Suecia, Malaui, Brasil- e, incluso, regiones - como la Comunidad Europea- y organizaciones — como la 
UNESCO - entre otros, han generado guías o directrices y principios éticos de la investigación en humanidades y ciencias sociales.

En Finlandia, por ejemplo, estas directrices y principios los dividieron en tres áreas: (1) respetar la autonomía de los participantes (sujetos) de la investigación, (2) evitar el daño y (3) privacidad y protección de datos (National Advisory Board on Research Ethics, 2009). En la guía de la Comisión Europea, cobra especial relevancia lo concerniente a cuestiones éticas sobre metodología, la protección de datos y privacidad, obtener el consentimiento informado, la estigmatización y discriminación (European Commission, 2010).

En el caso de México, Hernández (2016) señala la preocupación por el tema ético en el ámbito de las ciencias sociales y humanidades con el documento elaborado por la sección de Ciencias y Humanidades de la Academia Mexicana de las Ciencias en 2001 denominado Propuesta para el desarrollo de las ciencias sociales y las humanidades en México, sin embargo reconoce que son exiguos los esfuerzos universitarios para regular las prácticas éticas en la investigación social y humanística.

Así también, los estudios relacionados con la experiencia que los investigadores de ésta área han tenido, en cuanto a los procesos de revisión ética relacionada con estas guías y directrices, son escasos. Wynn et al. (2014) realizaron una encuesta en Australia acerca de la experiencia de investigadores con los comités de ética.

Los participantes correspondían a diferentes disciplinas: ciencia clínica, ciencias sociales y humanidades.

Otros estudios relativos al proceso de investigación - donde participan investigadores de humanidades - se relacionan con opiniones y hábitos de publicación, en Argentina (Bongiovani, Gómez, \& Miguel, 2012); nuevas tecnologías y redes sociales, en España (Baraibar \& Cohen, 2012).

Sin embargo, aunque existen trabajos relacionados con la experiencia de los investigadores en humanidades, en ciertos procesos de la investigación científica y la ética en algunos países, es escaso su estudio en México.

Con relación a esto, en el presente trabajo se plantea como objetivo general obtener la percepción, así como analizar e identificar las experiencias y prácticas de investigadores con grado de Doctor que se encuentren registrados con perfil SNI, en el área IV de Humanidades y Ciencias de la Conducta en México, respecto de situaciones éticas en la investigación propia de esta área. 
Por lo antepuesto, como parte de los objetivos específicos, se trata de conocer la percepción y opiniones que los investigadores tienen respecto de las necesidades de regulación ética y áreas de oportunidad de ética vividas en el proceso de recolección de datos primarios (encuestas, instrumentos, observación, experimentación, muestra y análisis de datos).

Asimismo, se busca identificar la tendencia del tipo de investigaciones (cuantitativas, cualitativas o mixtas) que, de acuerdo a su área de investigación, realizan; así como percibir conductas éticamente inaceptables o cuestionables que se suscitan en las publicaciones, respecto de la evaluación de artículos por editores y/o árbitros.

Además, se busca revelar situaciones de conflicto de interés que han vivido los investigadores, así como la percepción que se tiene de la situación actual de la ética en la investigación en México.

De esta manera, la relevancia de este proceso se distingue por lograr captar la percepción directa de estos investigadores, cuyo origen académico formativo, y en el que actualmente ejercen la labor de investigadores, es diverso entre ellos; lo que permite obtener la multiplicidad de experiencias vividas y percibir las áreas de oportunidad de la ética presente no sólo en el área de humanidades, sino en un contexto nacional en México.

\section{MATERIALES Y MÉTODOS}

Con el objeto de analizar las opiniones de los investigadores acerca de los aspectos éticos en los procesos de investigación en el área de humanidades, se establece un método de trabajo de tipo exploratorio de corte cualitativo; para ello, se plantean tres etapas principales, las cuales se describen más adelante.

El análisis cualitativo considerado en este trabajo se sostuvo a través del enfoque fenomenológico; para tal efecto, se tomó como base las experiencias individuales de los sujetos que conforman su muestra de estudio.

"Estos diseños son similares al resto de los que conforman el núcleo de la investigación cualitativa y, tal vez, aquello que los distingue reside en que la o las experiencias del participante o participantes son el centro de la indagación” (Hernández, Fernández y Baptista, 2015).

En tal sentido, Moustakas (1994), explica la importancia de profundizar en la comprensión de la experiencia. Para esto, se hace presente el objetivo del enfoque fenomenológico; el cual, 
consiste en conocer a detalle lo que el entrevistador identificó en su contexto, así como vivir su experiencia y sentir sus emociones; por lo que el sesgo personal o que el investigador percibe previo al estudio, puede ser reducido ampliamente. Willig y Stainton (2007), describen:

[..] En la medida en que Husserl fue el fundador de la fenomenología, quien en 1977 también reconoció explícitamente una versión pre-trascendental de su método, la fenomenología incluye todas las características de la tradición goetana (observación cuidadosa, descripción sin prejuzgar, uso de la intuición, etc.), más el uso de la reducción psicológica del fenómeno.

El uso de esa reducción significa que todo lo que se da experimentalmente al investigador se considera un fenómeno, simplemente una presencia sin añadir y que lo que está presente existe realmente en la forma en que está presente.

En tal sentido, Giorgi y Giorgi (2003) desarrollaron una metodología de investigación fenomenológica para orientar los estudios en esta área. Este método se ilustra en la siguiente figura:

Figura 1. El método de investigación fenomenológica existencial.

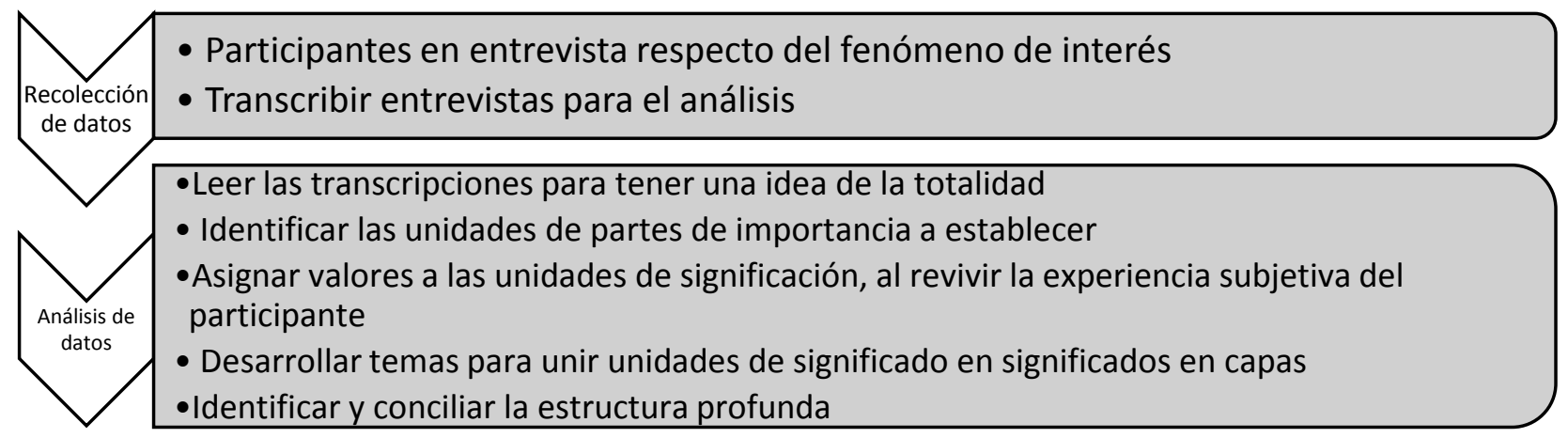

Fuente: Giorgi, A. y Giorgi, B. (2003). Phenomenology (tomado de: Anol Bhattacherjee, 2012). Social Science Research: Principles, Methods, and Practices, University of South Florida.

Al considerar la metodología citada, se detallan las fases realizadas en este proceso de investigación. Una primera etapa de investigación de gabinete consiste en exploraciones realizadas en el tenor de la indagación en el área de humanidades que permita sustentar y determinar el contexto de trabajo.

En la segunda etapa se recolectan los datos, en este caso, la aplicación de doce entrevistas semi-estructuradas efectuadas a doctores dentro del SNI del área IV de humanidades y ciencias de la conducta, las cuales fueron grabadas y transcritas para su correspondiente análisis. 
En la tercera etapa, se categorizaron las variables cualitativas usando el software Atlas.ti, en donde la información externada en las entrevistas se organiza por familias y se categorizan por códigos.

A partir de los resultados obtenidos y su consecuente organización, se efectúa el análisis de los resultados y la discusión de los mismos, con la finalidad de llegar a las conclusiones y propuestas del tema de investigación; tal como se observa en la siguiente figura.

Figura 2. Metodología
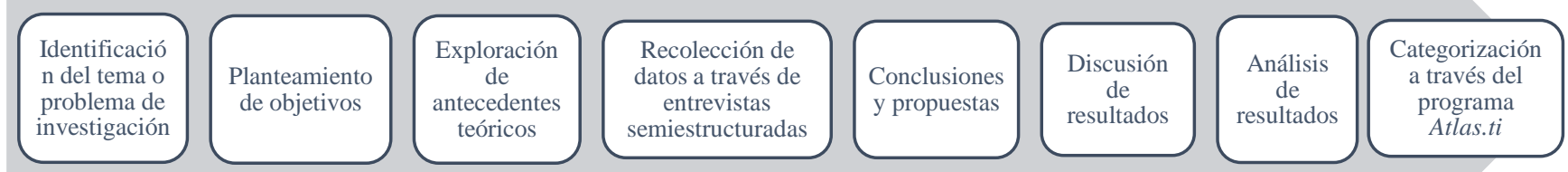

Fuente: Elaboración propia (con base en el procedimiento utilizado en la investigación).

Como se observa, una parte esencial de este método lo constituye la entrevista; de la cual, es recomendable que, previamente a su desarrollo, se elabore una plática entre el entrevistador y los entrevistados, a efecto de comprender el objetivo del tema abordado y forma de desarrollarlo; por ello, Thomas y Pollio (2002), sostienen que es de suma importancia aprender acerca de las presuposiciones del investigador, relativas a la naturaleza y el significado del fenómeno, y sensibilizarlo sobre cualquier situación que pudiera afectar al entrevistado.

Al momento de llevar a cabo una entrevista, el investigador (entrevistador) debe tener un amplio sentido de intuición, para retroalimentar el tema cuando exista el riesgo de que las respuestas obtenidas aborden una situación distinta al objetivo planeado; por lo que, continuando con lo expuesto por Thomas y Pollio (2002), las preguntas no se diseñan para conseguir una respuesta rápida ya conocida; del mismo modo, la cuestión fenomenológica no debe estar planteada para una explicación o declaración teórica; por lo que la entrevista se conduce hacia preguntas 
donde la persona entrevistada pueda describir sus experiencias de vida sobre alguna situación específica.

Mehra (2002), indica que una manera de obtener las opiniones de los entrevistados sin ideas preconcebidas o sesgos es unirse a uno mismo; por ello, resulta relevante — en este proceso — la exploración cuidadosa y a fondo, para lograr la conexión con los participantes del estudio.

\section{Recolección de datos}

Las entrevistas se llevaron a cabo en el último semestre de 2015, con duración de 15 a 30 minutos cada una. Se efectuaron de acuerdo al siguiente protocolo: en primer lugar, se hizo contacto inicial con el investigador al cual se le describió brevemente el objeto de estudio. Una vez que aceptó participar como entrevistado y previo al inicio de la sesión, se le pidió su consentimiento informado por escrito, así como su autorización para grabar la entrevista.

Posteriormente, ésta se inició siguiendo la guía de preguntas semi-estructuradas. Una vez concluida la entrevista, se procedió a realizar la transcripción de la misma.

Por su parte, Crabtree y Miller (1999), sostienen que un adecuado tamaño de la muestra siempre va a depender del tamaño de la población que se pretenda estudiar; ante ello, en primera instancia, debe realizarse un análisis en el cual sea posible que la muestra se integre de 5 a 20 casos de análisis.

Lo importante y sustancial consiste en que la selección pueda proporcionar información adecuada que facilite la toma de decisiones y sea representativa del contexto general de la población.

Asimismo, se encontraron diversas opiniones y estudios que hacen referencia a entrevistas semi-estructuradas. Para esto, Hernández et al. (2015), describen que este tipo de entrevistas, por su parte, se basan en una guía de asuntos o preguntas, y el entrevistador tiene la libertad de introducir preguntas adicionales para precisar conceptos u obtener mayor información acerca de los temas deseados (es decir, no todas las preguntas están predeterminadas).

Una vez realizadas las entrevistas, se procedió a categorizar los datos obtenidos de éstas, a través del software Atlas.ti; primero, desarrollando una matriz de datos en Excel (que funciona 
como archivo de acopio de la información vertida de las entrevistas semi-estructuradas) donde se colocaron símbolos a las columnas que permiten identificar cuáles son datos generales; se señalaron las columnas para cruzar información, así como las columnas referentes a la información básica a analizar y categorizar.

El archivo en Excel se exportó al sistema Atlas.ti, cifrándose los resultados a través de códigos de análisis de la información, con la intención de poder examinar los resultados con base en la teoría y el enfoque de la investigación.

\section{Categorización}

Para mantener un orden de la información obtenida de las entrevistas, se organizaron las preguntas por familias generales. Con base en esas categorías de familias, se identificaron códigos de análisis deductivos; tal como se muestra en la Tabla 1

Tabla 1. Categoría de análisis.

TABLA DE CATEGORÍAS DE ANÁLISIS

\begin{tabular}{l|l}
$\begin{array}{l}\text { CATEGORÍAS DE FAMILIAS } \\
\text { TIPOS DE INVESTIGACIÓN }\end{array}$ & $\begin{array}{l}\text { Cualitativa } \\
\text { Cuantitativa } \\
\text { Mixtas } \\
\text { Cómo los conoció }\end{array}$ \\
PRINCIPIOS DE ÉTICA & $\begin{array}{l}\text { Dónde los conoció análisis } \\
\text { Procedimientos metodológicos } \\
\text { Conocimiento informado } \\
\text { Plagio }\end{array}$ \\
APLICACIÓN DE LOS & Validación de los instrumentos \\
PRINCIPIOS ÉTICOS & $\begin{array}{l}\text { Falsedad en los datos } \\
\text { Datos sin recolección }\end{array}$ \\
ÉTICA EN LA RECOLECCIÓN datos \\
DE DATOS & $\begin{array}{l}\text { Fiabilidad en el análisis de dation } \\
\text { Datos incompletos }\end{array}$ \\
ÉTICA EN LA MUESTRA Y & Condiciones inadecuadas en el muestreo \\
ANÁLISIS DE DATOS & Respeto a los participantes \\
Control de los equipos de responsables & Plagio en la publicación \\
Ética en la citación \\
Reproducción de publicaciones \\
ÉTICA EN LAS & Evaluación laxa \\
PUBLICACIONES & Evaluación severa \\
Preferencias en la edición \\
Plagio en la edición \\
Anonimato en la evaluación \\
ÉTICA EN EDITORES Y/O \\
ÁRBITROS & Financiamiento
\end{tabular}




\begin{tabular}{|c|c|}
\hline & $\begin{array}{l}\text { Conflictos económicos } \\
\text { Conflictos personales } \\
\text { Conflictos políticos } \\
\text { Crédito a la colaboración }\end{array}$ \\
\hline $\begin{array}{l}\text { ÉTICA EN LA } \\
\text { INVESTIGACIÓN EN MÉXICO }\end{array}$ & $\begin{array}{l}\text { Reglas del CONACyT } \\
\text { Rigor en la investigación } \\
\text { Pérdida de capital intelectual } \\
\text { Burocracia } \\
\text { Pobre formación en investigación } \\
\text { Falta de apoyos } \\
\text { Red de colaboración }\end{array}$ \\
\hline
\end{tabular}

Fuente: elaboración propia (con datos de entrevista semi-estructurada).

Las respuestas ofrecidas en las entrevistas, al ser revisadas - literalmente - se relacionaron con la información, con cada uno de los códigos determinados; y, posteriormente, se procedió a la realización de una red semántica con el apoyo del software Atlas.ti. Ello permitió una mirada global a las relaciones entre categorías y códigos, según se puede observar en la siguiente figura 3. 
Figura 3. Red semántica de los datos obtenidos de entrevistas en el contexto ético en las humanidades.

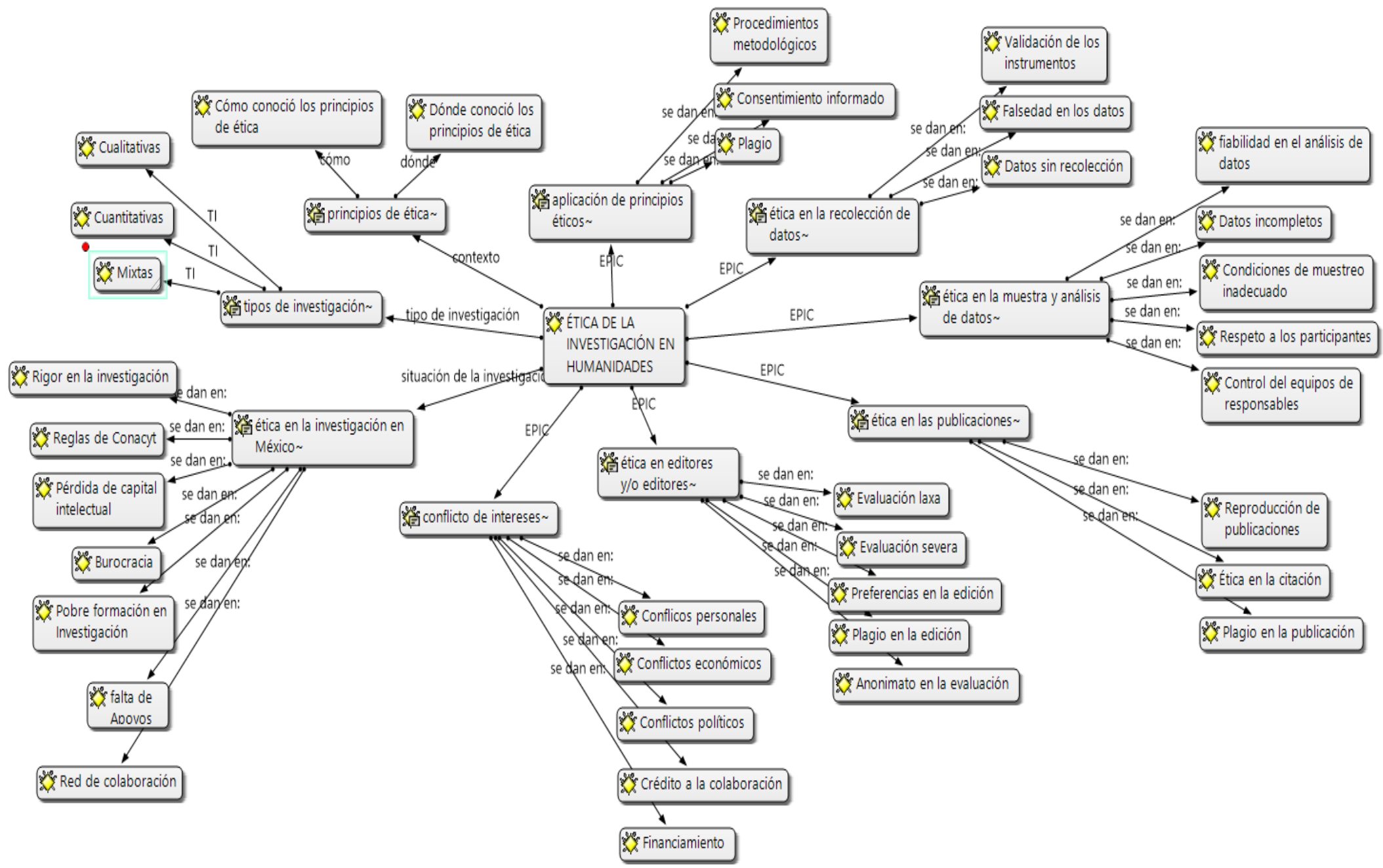

Fuente: Elaboración propia (con apoyo del software de Análisis de Datos Cualitativos Atlas.ti). 


\section{RESULTADOS}

La aplicación de doce entrevistas semi-estructuradas a doctores del SNI del área IV de humanidades y ciencias de la conducta, nos presenta la siguiente caracterización sociodemográfica: en cuanto a las disciplinas que cultivan, 6 no mencionaron, 4 en psicología, 1 en historia y 1 en educación. Del año en que obtuvieron el SNI por primera vez, el 75\% con menos de cinco años de haber ingresado: 5 en 2015, 1 en 2013, 3 en 2012 y del resto, 1 en 2010, 1 en 2006 y 1 en 2005. Y muy probablemente debido a su reciente ingreso, el 50\% ostenta el nivel 1 y del resto, sólo 1 en nivel 2, dos como candidatos y 3 no mencionaron.

En la figura 4 se concentran los resultados relativos a la tendencia del tipo de investigaciones que realizan, a sus experiencias con los principios éticos, así como el reconocimiento de sus primeros contactos con los principios éticos.

Figura 4. Resultados de entrevistas (parte I).

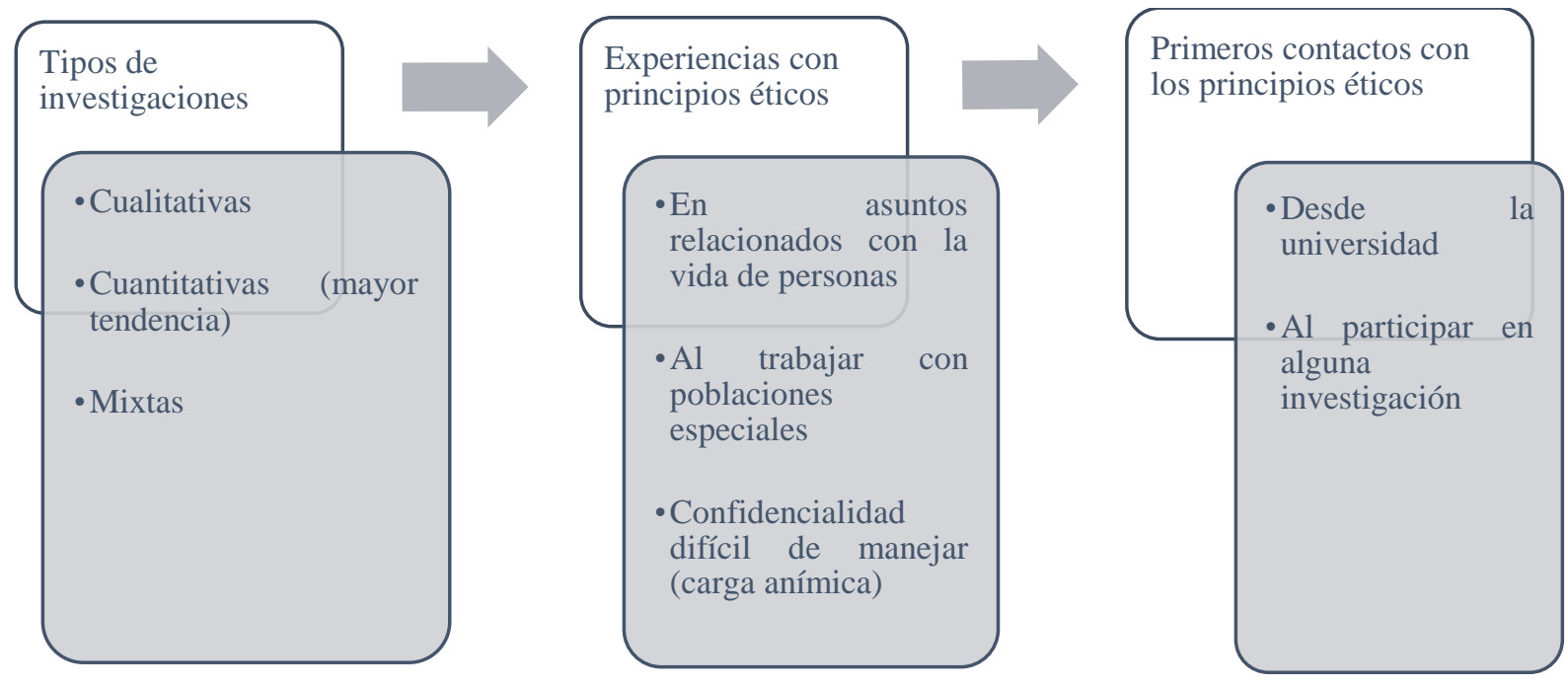

Fuente: Elaboración propia (con datos obtenidos de las entrevistas a investigadores SNI).

Por otra parte, en la figura 5, se concentran conductas éticas inaceptables que conocieron o vivieron los investigadores entrevistados como parte de su experiencia en el andar de la investigación académica en México. 
Figura 5. Experiencias reales de conductas éticas inaceptables en investigadores.

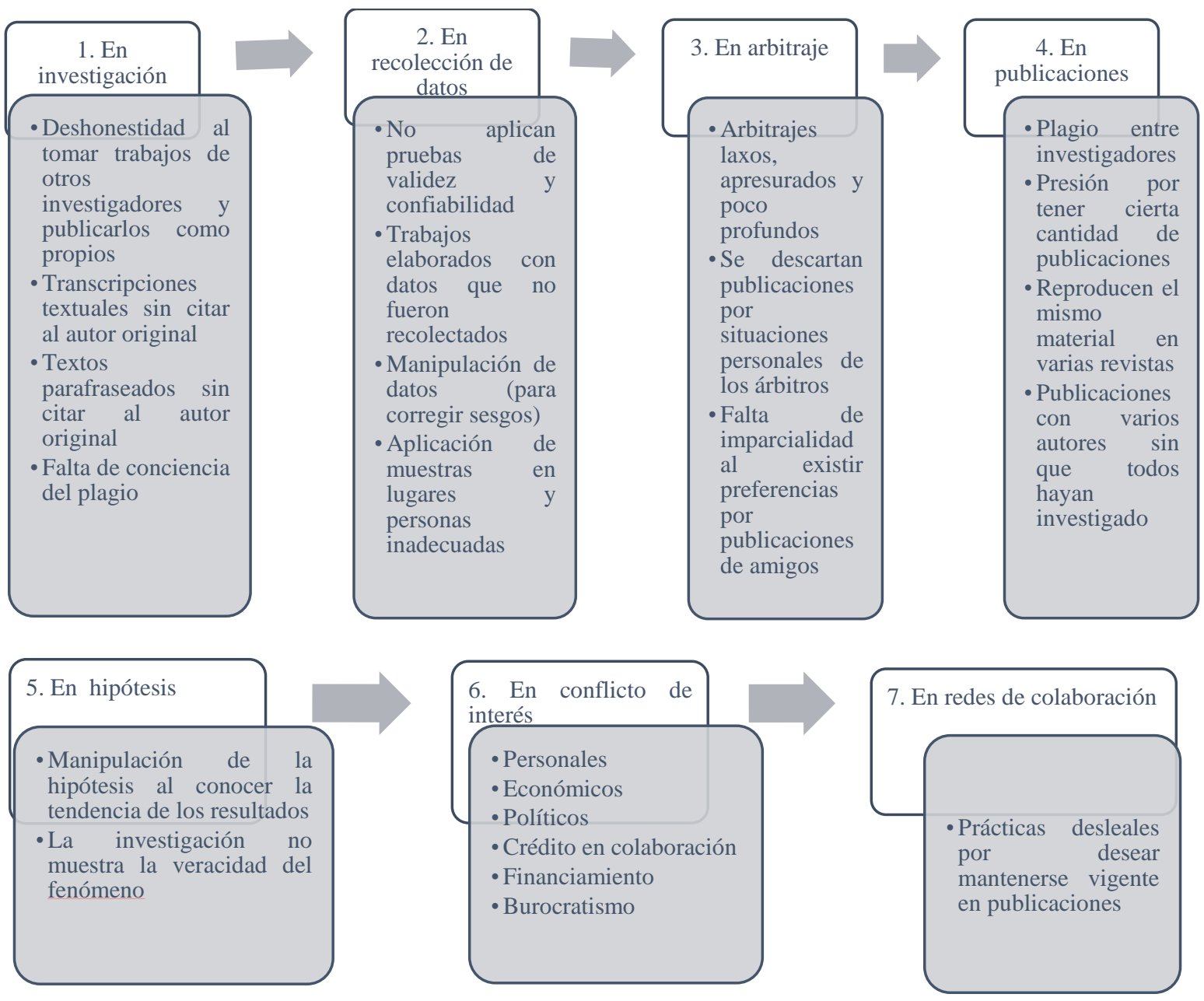

Fuente: Elaboración propia (con datos obtenidos de las entrevistas a investigadores SNI).

\section{DISCUSIÓN}

Con base en la información recabada y el análisis de las opiniones vertidas por los investigadores del área de humanidades, se procedió a la discusión de los resultados; bajo el entendido de que la regulación ética permite disminuir el riesgo de daño o atropello a la dignidad de las personas, la responsabilidad profesional de los investigadores y de las instituciones colaboradoras como base para proteger efectivamente a los participantes (Gaudlitz, 2008).

$\mathrm{Al}$ conocer las experiencias de los investigadores entrevistados respecto de sus experiencias éticas en la investigación, éstos coinciden en la necesidad del establecimiento de normas éticas más directas a su área, las cuales vigilen y sancionen el proceso metodológico desde la propia recolección de datos hasta el producto terminado que conlleva a publicar; ya que, todos ellos 
manifiestan vivencias de conductas éticas inaceptables en este proceso, cuyo detalle se presenta en el apartado de resultados. Ahora bien, como señala Cegarra (2012):

[...] los principales aspectos éticos del investigador abarcan la honestidad, admisión del error, lealtad, humildad; por lo que al conocer las situaciones de conductas éticas inaceptables vividas por algunos investigadores, éstos mostraron tener implícitos dichos valores éticos como parte de su formación personal y profesional y por ende, mostraron rechazo hacia las faltas éticas realizadas por otros colegas.

En ese sentido, en el libro titulado "Principios de ética, bioética y conocimiento del hombre" de Morales J., Nava G., Esquivel J. y Díaz L., (2011) se pretenden sentar las bases para la conformación de un comité de ética en la investigación en ciencias de la conducta, humanidades y ciencias sociales, justificando que en México, si bien los códigos de ética en estas áreas no están sujetos a una regulación legal, todos los comités deberían de estarlo, a efectos de ser capaces de regular las prácticas y las interacciones entre investigadores y participantes, a favor del beneficio mutuo.

Por su parte Guerriero, I. \& Magalhaes, M. (2015), en una investigación realizada en Brasil, difieren del carácter rígido desarrollado en las áreas de la medicina, haciéndose énfasis en dicha investigación en la necesidad de diseñar reglas flexibles en las investigaciones de carácter social, para que los comités realicen aportaciones que faciliten el desarrollo de la investigación, así como reforzar los resultados obtenidos.

Sobre los primeros contactos que los entrevistados tuvieron con los principios éticos y morales algunos mencionan que fue al participar directamente en alguna investigación o durante sus estudios oficiales (propiamente en la etapa universitaria, cuando se adentraron a los procesos de investigación).

Pues desde que yo estaba en la licenciatura, en materias de seminario de investigación, yo recuerdo haber conocido normas éticas que regulaban la investigación (participante 3).

En forma más específica, los entrevistados indicaron en qué aspectos aplican los principios éticos, considerando importante indicar que la ética se aplica desde el momento mismo de plantear el problema; por lo que una falla en los procesos metodológicos también es un problema ético, por no atender adecuadamente los lineamientos de investigación. 
Hernández (2016) señala que los investigadores adoptan decisiones éticas que entrañan ciertos compromisos morales que exigen la confiabilidad y el respeto a los principios que norman el devenir cotidiano y las relaciones entre los miembros de la comunidad científica y académica.

Por otro lado, refieren la importancia de solicitar un consentimiento informado por parte de los sujetos que participan en la investigación a fin de mantener su permiso para investigar y el derecho a su confidencialidad.

Sobre todo, conseguir el consentimiento de los participantes; porque, en algunos casos, son menores de edad y hay que conseguir el consentimiento de los padres (participante 7).

El sentir de los derechos humanos de los participantes en las investigaciones siempre debe estar presente; y, como señala Vanegas (2010), para mostrar los principios éticos de los derechos fundamentales, es necesario visibilizar la urgencia del reconocimiento de los seres humanos como fundamento y esencia del derecho.

Otros de los problemas percibidos en la aplicación de los principios éticos se refieren a la falta de honestidad de algunos investigadores, al retomar trabajos de otros autores como si fueran propios y publicarlos en su nombre.

Con esa misma intención, se observan faltas de ética en el proceso de citación, transcribiendo espacios textuales sin el adecuado uso de comillas o parafraseando información sin anotar la cita del trabajo original.

Un error en una parte de citado era reprobado automáticamente; y, entonces, uno aprende que si no citas y das crédito a las ideas de otro, eso es un error grave y si se demuestra que es plagio, pude haber expulsión (participante 2).

Con respecto a la aplicación de la ética en la recolección de datos, los entrevistados manifestaron que ello consiste en principios que deben seguirse durante todo el proceso de investigación científica; y muy especialmente durante la recolección de datos, puesto que de éstos dependerán los resultados de la investigación realizada.

En este sentido, exponen que a un instrumento de recolección de datos se le deben aplicar pruebas que demuestren validez y confiabilidad, pues de ello dependerá la legitimidad y confianza en la información obtenida. Sin embargo, en algunos casos, se aplican instrumentos sin el debido proceso de validación, lo que conlleva una falta al proceso de investigación y a la ética en la manipulación de resultados. 
En los instrumentos hay dificultades en la validación o que, tal vez, dentro del país no se ha utilizado el instrumento; entonces, hay que trabajar en toda la validación de éste (participante 7).

Asimismo, mostraron su interés por las prácticas no éticas de algunos investigadores que desarrollan trabajos de investigación con datos que no fueron recolectados en la realidad, o que fueron manipulados falsamente. De igual manera, son relevantes los comentarios cuando expresan que han llegado a proseguir con investigaciones aun cuando se observa en el desarrollo que la información que están proporcionando es falsa, incompleta o con sesgo.

[...] cuando llegamos a la fase de la prueba piloto, me he dado cuenta que algunos estudiantes contestan el cuestionario sin interés y que no exponen la realidad [...] (participante 8).

Lo anterior, pone de manifiesto cómo los investigadores son conscientes de los problemas asociados con el proceso de investigación, así como errores y faltas de ética en dicho procedimiento; sin embargo, deciden continuar con el proyecto, presentando resultados que pueden ser erróneos o manipulados en aras de la aceptación de las hipótesis planteadas.

Diversos investigadores coinciden con lo discutido en esta investigación, como San Martín (2007), quien aborda lo relacionado con la preocupación ético-procedimental en la investigación educativa o con alcance indirecto sobre problemáticas de carácter moral; a la vez, refleja en sus conclusiones de manera panorámica una penuria de aspectos éticos, resaltando lo escaso de exponer las limitaciones y los cuidados que todo investigador debe procurar respetar antes y durante el desarrollo de cualquier investigación.

En lo concerniente a la ética en la muestra y análisis de datos, los entrevistados mostraron su preocupación a los problemas éticos en la recolección de los datos, en situaciones o condiciones externas que afectaban el proceso de muestreo; por ejemplo: lugares inadecuados para allegarse de la información, sujetos que no corresponden a los establecidos en la muestra, momentos y lugares inadecuados para aplicar el muestreo y que resulta de información falsa o sesgada. 
Desde el ámbito jurídico, Hernández, M. (2016) destaca algunas de las malas prácticas, o de comportamientos contrarios a la ética que se dan en la investigación, las cuales coinciden con las manifestadas por los entrevistados para el presente estudio, enumerando además algunas consecuencias de estos actos.

En otras ocasiones - indicaron los entrevistados - los datos son incompletos o no son fiables en su obtención, al ser manipulados con la intención de que responda a la hipótesis planteada y no que refleje la veracidad del fenómeno.

Muchas veces no te sale el análisis como tú lo esperabas, entonces, entra conflicto cuando no da un resultado y están en si quitas un pedazo de la muestra o no, para que salga el análisis bien. En si lo muevo, porque no me está saliendo como esperaba, pero qué tal si manipulas eso de alguna manera para que salga bien. Pero es algo que la población está diciendo, entonces, no se puede manipular a como pienso yo que son las cosas (participante 11).

En ese sentido, Aranguren (2008), señala que en el trabajo de campo, se afronta la ética a través de escuchar a los entrevistados y enfrentar a la vez los silencios de éstos en situaciones demarcadas; por lo que concluye que la ética en estos casos no puede ser regulada por mecanismos de normalización.

De la misma forma, los entrevistados comentaron que las investigaciones, preferentemente en los estudios de corte cualitativo, se operan situaciones personales difíciles de manejar por la carga anímica y sensible de la información; en donde los principios éticos de respeto a los participantes, la confiabilidad y el anonimato son aspectos a cuidarse durante la recolección de los datos.

[...] En una ocasión estábamos tratando indicio de intento suicida en jóvenes, [...] muchas veces las personas se sensibilizan y exponen sus propias experiencias. Y es fuerte hablar del tema del suicidio; y hay personas que, de repente, sueltan el llanto..., pero, pues, todos estamos ahí para que lo que ahí se escucha ahí se queda, que nunca se dirá el nombre de la persona. Es totalmente confidencial [...] (participante 12).

En algunas partes la investigación puede verse limitada en sus resultados; ello, debido a la naturaleza del tema o a la ética profesional, que depende de cada individuo; por lo mismo, se entiende que los resultados no pueden interpretarse en forma general para los posteriores casos. Beaucham (2003) comenta respecto de sus propias investigaciones: 
¿Es posible predecir el resultado en los casos éticos, dado que las diversas morales pueden no ser universales? Tal vez aún más importante, ¿es posible, a través de la comprensión de las experiencias [...] en los casos específicos investigados [...] se puede ayudar a otros que pueden experimentar experiencias similares?

Con respecto a la ética en las publicaciones, los entrevistados mostraron su reprobación a la práctica del plagio y a las posibles consecuencias de castigo con la expulsión de los investigadores del Sistema Nacional de Investigadores en México. Pero también la consideran como una práctica a la que se ven obligados muchos investigadores por la necesidad de publicar un cierto número de artículos en determinado periodo.

Por esta razón, se llegan a transgredir los principios de ética, reproduciendo sus propias publicaciones en varias revistas, retomando material trabajado y elaborando un nuevo artículo sobre esta base. Otros de sus colegas se van "apadrinando", trabajando en una especie de red de colaboración; en donde una persona publica una ponencia con varios coautores que no hicieron aportaciones importantes al trabajo, pero que a otro integrante del grupo le tocará publicar, aplicando el mismo proceso de acompañamiento; todo esto con el objeto de acrecentar el número de publicaciones.

[...] Se nos obliga a tener una trabajo a destajo en donde hay que publicar, hay que publicar [...] cuando nos dicen de revistas indexadas de reconocido prestigio, entonces, allí mucha gente, induce a lo que son llamados refritos de una investigación, se saca un capítulo, saca otro capítulo, saca una ponencia, saco una conferencia [...] (participante 4).

Es de llamar la atención lo mencionado en los párrafos precedentes, donde se hace alusión a la "obligación de trabajar a destajo con el objeto de acrecentar sus publicaciones", para poder mantenerse en cierto nivel o estatus que, como investigadores, se rigen bajo categorías normativas establecidas a nivel nacional en México; percibiéndose, de alguna manera, cierta justificación por atender el sistema; tal circunstancia hace evocar lo siguiente: "Para todas las acciones tomadas por las personas, cuanto más a menudo una acción particular de una persona es recompensada, es más probable que la persona vuelva a realizar esa acción” (Homans, 1974).

En este sentido, se emplea la teoría de Homans, la cual se basa en el principio de la recompensa. En una organización gubernamental es común que existan ciertas recompensas, las cuales están enfocadas a premiar el comportamiento de los individuos que se apegan a determinada 
norma; de tal manera que dicha situación es imitada por las demás personas que desean también obtener el beneficio de la recompensa.

En lo que Drucker denomina "la prueba del espejo", cita lo siguiente: "Lo que la ética requiere es preguntarse: ¿Qué tipo de persona quiero ver cuando me afeito por la mañana? La ética, en otras palabras, es un sistema de valores claro" (Drucker, 1999).

En analogía con lo expuesto, es de gran trascendencia que las personas que laboran en la organización, tengan también una ética personal que encuadre con el objetivo. "Para ser eficaces en una organización, los propios valores deben ser compatibles con los valores de la organización, no tienen que ser los mismos, pero deben estar lo suficientemente cerca como para que puedan coexistir" (Drucker, 1999).

Lo mismo sucede en las organizaciones educativas, en donde el quehacer universitario contempla la generación y aplicación del conocimiento mediante la investigación; por lo que sus investigadores deben tener un comportamiento ético y alineado con las políticas institucionales (locales y nacionales).

Sin embargo, existen varias opiniones respecto de los resultados que se obtienen en las organizaciones de cualquier índole; los cuales, en ocasiones, distan mucho de ser los esperados. Ante esta situación, De Colle y Werhane (2008), manifiestan:

[...] Debe haber una manera de asegurar que las personas dentro de una organización sean responsables de su falta de ética; especialmente si su propia agenda personal se utiliza para dañar a otros dentro de la organización. Los individuos poco éticos siempre se encontrarán en todos los sistemas e industrias. Finalmente, si los valores personales de los individuos dentro de una organización eclipsan verdaderos valores éticos, la organización sufrirá.

En la mayoría de los casos, es común encontrar conductas que se desvían o, en su caso, son contrarias a los códigos de ética de las organizaciones gubernamentales.

De Colle y Werhane (2008), sostienen que puede haber muchas razones para que aquellos en el poder no se adhieran a estos códigos. Tal vez, se debe a la falta de temor al castigo, a un manejo inapropiado de liderazgo, ausencia de capacitación, a la protección de los demás en la organización o, incluso, a que no se les dé o enseñen estas habilidades.

Continuando con la discusión de los resultados, se describe la etapa donde la investigación se ha realizado y se encuentra en proceso de publicación. 
Es aquí donde los entrevistados externaron su opinión acerca de problemas éticos en la etapa de revisión por editores y/o revisores.

En general, consideran que el rigor en la revisión depende del prestigio de la revista; si ésta cuenta con factor de impacto del Journal Citation Reports (JCR), entonces, la evaluación es más estricta y con apego a los lineamientos metodológicos y éticos de todo proceso de investigación científica.

Ahora bien, cuando se trata de revistas de menor nivel y rigor académico, los problemas éticos se observan en la revisión superflua o laxa de los requisitos de investigación.

También tienden a ser más severos en la evaluación de trabajos de corte cualitativo que cuantitativo; o se dejan llevar por el nombre de los autores de un artículo, aun suponiendo que debe hacerse a "doble ciego" o en forma anónima, por lo que se muestran problemas éticos al mostrar preferencias en la revisión de los escritos.

Asimismo, pueden verse obligados a una revisión poco profunda debido a los tiempos con que se envían los trabajos para sus dictámenes.

Otro aspecto que se debe cuidar durante este proceso es la adecuada referencia de artículos durante la citación; puesto que, llegado el momento de evaluar los trabajos en vías de la publicación, se hacen uso de programas informáticos que detectan plagios con relativa facilidad, evidenciando faltas de ética durante este proceso.

Para un congreso, me pidieron ayuda para dictaminar algunas ponencias, y quince días antes de la publicación de los dictámenes me envían como doce artículos; me quedé con la idea de que no cumplí del todo con aquella tarea, porque ante la prisa, no hice una buena lectura; y, por lo mismo, los dictámenes pueden ser muy laxos (participante 8).

Durante todo el proceso de investigación pueden ocurrir "conflictos de intereses"; pero, sobre todo, cuando las investigaciones se encuentran finalizadas y los resultados suelen no ser acordes con algunos grupos o intereses políticos y económicos.

Los entrevistados expresaron que aparecen estos problemas cuando al organismo que financia una investigación suelen parecerle demasiado rígidos los resultados, por lo que los prefieren menos comprometidos y por ello solicitan "matizarlos", por cuestiones de intereses políticos. 
En otras ocasiones, se crean conflictos personales, por no otorgarse el debido crédito a la colaboración de los trabajos o negárseles la publicación porque muestran inconsistencias en el desarrollo, entre otras posibilidades.

[...] Porque es mi amigo lo voy a hacer de una manera, va a privar la amistad y no la rigurosidad académica, si yo veo que la realidad es ésta, voy a matizarla para que no se enoje quien la financia; y lo peor: llegar al plagio vil... todo tan comercializado que creo también le ha llegado a la compra de la ética (participante 4).

En general, los entrevistados tienen una opinión dividida con respecto a la situación de la ética de la investigación en México. Por un lado, consideran que se está haciendo un trabajo más profundo por parte del Consejo Nacional de Ciencia y Tecnología (CONACyT), para evitar problemas en la publicación de resultados, manteniendo un mayor rigor en la evaluación de trabajos de investigación; pero consideran que faltan apoyos para permitir el desarrollo de la investigación en todos los ámbitos, pues consideran que en el área de humanidades no se da el mismo apoyo que el que ocupan otras ramas de las ciencias (como las ingenierías o ciencias naturales).

Sobre este tema, Romero (2013), resalta en su investigación que organismos de ciencia y tecnología -de otros países-, disponen como requisito para el financiamiento de la investigación que se debe contar con un aval por parte de un comité de ética de instituciones de educación superior inclinado a las ciencias biomédicas; concluyéndose en dicho estudio que de alguna manera, las investigaciones sociales cumplan con tal requisito, preguntándose entonces si se están copiando modelos propuestos por las ciencias biomédicas como si fuese lo mismo que las ciencias sociales.

En contraste a lo anterior Wynn (2016) evidencia otra apreciación, concluyendo que los comités de ética han expandido su jurisdicción, forzando a que investigaciones sean valoradas por dichos comités, obteniendo como resultado una apremiante inconformidad, es decir, investigadores del área de las ciencias sociales y humanidades creen que los comités de ética son perjudiciales para la investigación de calidad y no creen que los cambios requeridos por dichos comités protejan a sus participantes en la investigación.

Otra situación de la ética de la investigación en México los entrevistados consideran que se sigue manteniendo un problema burocrático en los procesos de selección de candidatos al Sistema Nacional de Investigadores, que obstaculiza la entrada de nuevos investigadores y mantiene — a 
los que están dentro del sistema — a prácticas de publicación rígidas que, en ocasiones, son difíciles de cumplir.

Por otro lado, expresan su desazón por la pobre formación en investigación en los estudiantes universitarios; tiene que trabajarse en esta área de oportunidad para incrementar los niveles de investigación en México. En este sentido, González (1995) ilustra:

[...] para encontrar el significado actual de la palabra Humanismo e intentar un proyecto posible, no basta una disciplina con denominación abstracta y hasta visos de irrealidad fáctica, sino que se necesitan tres elementos esenciales y un proceso forzoso. Los elementos son: un contenido que, indubitablemente, incorpore las reflexiones primeras sobre el ser humano y su acontecer, un educador convencido de lo que hace y un alumno que tenga ganas de compartir con él la dura tarea de empezar todos los días; el proceso es paideútico, pues sólo la educación puede encarar provechosamente la tarea de la recreación constante.

Por último, los investigadores entrevistados desaprueban la práctica de redes de colaboración, porque resulta que por el hecho de mantenerse en publicaciones se produce una red de acompañamiento desleal y de falta de ética. No obstante, concuerdan que no es un problema sólo de México, sino de la investigación misma a nivel mundial.

Los derechos humanos se encuentran como parte del proceso implícito de la ética en las humanidades y, en este sentido, Ferrero (2000) señala que la Declaración Universal de los Derechos Humanos, aprobada por la asamblea de la Organización de las Naciones Unidas (el 10 de diciembre de 1948), incluye una parte de interés general con relación a la defensa de la dignidad de las personas.

Finalmente, se resume la necesidad de requerimiento de consideraciones legales específicas con las particularidades de la transdisciplinariedad; por lo que es de relevancia asentar que en México existe - entre otros- un instrumento normativo relevante para la aplicación de la ética en investigación para temas bioéticos, como lo es la Guía Nacional para la integración y Funcionamiento de los Comités de Ética en Investigación, emitido por la Comisión Nacional de Bioética (2016), actualizado y con enfoque multidisciplinario para enriquecer el análisis de los dilemas que se suscitan, principalmente en el campo de la biomedicina y el desarrollo de políticas públicas, con un enfoque incluyente y perspectiva de género, en beneficio de la población. 


\section{CONCLUSIONES}

El presente estudio permitió descubrir las experiencias vividas por los investigadores en una realidad que actualmente existe en los procesos de investigación desde la perspectiva de la ética y las humanidades; haciéndose necesario impulsar cambios desde nuestros distintos ámbitos de conocimiento hacia un preponderar ético en nuestro quehacer investigativo, percibiéndose la ética en ocasiones y, desafortunadamente, en un segundo plano.

Aunado a esto, resalta la necesidad — que es de gran relevancia— de la expansión, creación y difusión de normas éticas hacia otras áreas de conocimiento; ya que, cuando se refiere a procesos de investigación que implica trabajar con seres vivos (y no en todos los casos), éstos se autorizan a través de los comités de ética, estando ausentes y no creados estos comités para áreas específicas en humanidades y ciencias sociales (al menos en México).

En este sentido, los entrevistados mostraron su reprobación a la práctica del plagio y a la necesidad de consecuencias de castigo con la expulsión de los investigadores del Sistema Nacional de Investigadores en México.

No obstante lo anterior, la presente investigación no sólo permitió afirmar situaciones ya conocidas de manera informal en el ámbito de la investigación, sino que, además, permitió identificar áreas de oportunidad que permitan la creación de políticas y códigos éticos adaptadas a las distintas áreas de conocimiento; las cuales vigilen y sancionen el proceso metodológico desde la propia recolección de datos hasta el producto terminado que conlleva a publicar. Esto permitirá truncar, paulatinamente, las mencionadas conductas éticas inaceptables.

La sensatez de Nussbaumcitada (en García, 2012), hace ver la ausencia de la formación humanística:

[...] esta crisis da cuenta de la tan relevante ausencia de una formación humanística seria y contundente. Aun a sabiendas que las humanidades han transformado el mundo en el que vivimos y han desempeñado un papel fundamental en el proceso de la formación sociopolítica - a grado tal que han apuntalado el desarrollo de la democratización de algunos países- la sociedad en general se preocupa de manera principal en prepararse casi únicamente para una futura prosperidad económica, centrándose en la ambición de tener ingresos. Esto sucede en todos los niveles, desde los individuales hasta los estatales y es una cuestión mundial. 
En general, la ética es vista de varias formas, según el país en que se investigue; y, como señala McNeill (1989): "Una de las distinciones obvias entre Estados Unidos y otros países es el grado de control gubernamental sobre el proceso regulador de la revisión ética, aparte de los factores históricos, culturales y políticos"; por lo que este grado de control en México se refleja endeble, donde los investigadores "juegan y/o se adaptan al sistema". 


\section{REFERENCIAS}

AMM, D. d. (2016). Principios éticos para las investigaciones en seres humanos. Recuperado el 30 de octubre de 2015 en: http://www.wma.net/es/30publications/10policies/b3/

Aranguren, J. (2008). El investigador ante lo indecible y no inenarrable (una ética de la escucha). Revista Nómadas, 29, 20-33.

Área IV SNI (2016). Criterios específicos de evaluación del área IV humanidades y ciencias de la conducta del Sistema Nacional de Investigadores. Recuperado el 11 de diciembre de 2016 de http://conacyt.gob.mx/index.php/el-conacyt/sistema-nacional-de-investigadores/marcolegal/criterios-sni/830-criteriosespecificosareaiv/file

Baraibar, Á. y Cohen, S. (2012). Nuevas tecnologías y redes sociales en la investigación en Humanidades. La Perinola, (12), 155-164.

Beauchamp, T. L. (2003). A defense of the common morality. Kennedy Institute of Ethics Journal, 13 (3), 259-74.

Bongiovani, P., Gómez, N. y Miguel, S. (2012). Opiniones y hábitos de publicación en acceso abierto de los investigadores argentinos. Un estudio basado en los datos de la encuesta SOAP. Revista Española de Documentación Científica, 35(3), 453-467.

Cegarra, J. (2011). Metodología de la investigación científica y tecnológica. Madrid, España: Ediciones Díaz de Santos.

Comisión Nacional de Bioética. (s/a). Código de Nuremberg. Normas éticas sobre experimentación en seres humanos. México: Comisión Nacional de Bioética. Recuperado el 1 de noviembre de 2015, en: http://www.conbioetica-

mexico.salud.gob.mx/descargas/pdf/normatividad/normatinternacional/2.INTL._Cod_Nuremberg.p df

Comisión Nacional de Bioética. (s/r). Informe Belmont. Principios y guías éticos para la protección de los sujetos humanos de investigación. National Institutes of Health. Recuperado el 05 de noviembre de

2015, en: http://www.conbioetica mexico.salud.gob.mx/descargas/pdf/normatividad/normatinternacional/10._INTL_Informe_Belmon t.pdf

Crabtree, B.F. y Miller, W.L. (1999). Doing qualitative research (2nd Ed.). Thousand Oaks CA: Sage.

Cribb, R. (2004). Ethical regulation and humanities research in Australia: problems and consequences. Monash Bioethics Review, 23(3), 39-56.

De Colle, S. y Werhane, P.H. (2008). Moral motivation across ethical theories: What can we learn for designing corporate ethics programs? Journal of Business Ethics, 81(4), 751-764.

Drucker, P.F. (1999). Management challenges for the 21st century. New York, NY: HarperCollins. 
European Commission. (2010). Guidance Note for Researchers and Evaluators of Social Sciences and

Humanities Research. Recuperado el 10 de marzo de 2016, en: http://ec.europa.eu/research/participants/data/ref/fp7/89867/social-sciences-humanities_en.pdf

Ferrero, A. (diciembre de 2000). La ética en psicología y su relación con los derechos humanos. Fundamentos en Humanidades, 1(2), 17-31.

García, D. E. (2012). Reseña de sin fines de lucro. Por qué la democracia necesita de las humanidades de Martha Craven Nussbaum. Revista Enfoques: Ciencia Política y Administración Pública, X (16), 181185 .

García, R. (1970). Origen, significado y crítica de las humanidades. Encuentro: Revista Académica de la Universidad Centroamericana, 31-44.

Gaudlitz, M. (2008). Reflexiones sobre los principios éticos en investigación biomédica en seres humanos. Revista Chilena de Enfermedades Respiratorias, (24), 138-142. Recuperado el 10 de noviembre de 2015, en: http://www.scielo.cl/pdf/rcher/v24n2/art08.pdf

Giorgi, A. y Giorgi, B. (2003). Phenomenology. En: J. A. Smith (Ed.) Qualitative Psychology: A Practical Guide to Research Methods. London: Sage Publications.

González de Tobía, A. M. y Verde, C. V. (1995). Significaciones y perspectivas del humanismo: Humanismo y Humanidades, (2), 107-135.

Guía Nacional para la Integración y el Funcionamiento de los Comités de Ética en Investigación. Quinta edición (2016). Secretaría de Salud/ Comisión Nacional de Bioética.

Hernández M., M. del P (2016). Ética de la investigación. En Javier Saldaña Serrano (Coord), Ética Jurídica (Segundas Jornadas) (pp. 3-9). México: UNAM, Instituto de Investigaciones Jurídicas. Recuperado el 11 de diciembre de 2016 de https://archivos.juridicas.unam.mx/www/bjv/libros/8/3970/3.pdf

Hernández, R., Fernández, C. y Baptista, M. (2015). Metodología de la investigación. (5ta. Ed.) México: McGraw Hill.

Homans, G.C. (1974). Elementary forms of social behavior. New York, NY: Hardcourt Brace Jovanovich. Husserl, E. (2002). Renovación del hombre y de la cultura. México: Anthropos. Recuperado el 28 de octubre de 2015, en: https://fenomenologiaymundo.files.wordpress.com/2014/08/edmund-husserlrenovacion del-hombre-y-de-la-cultura-cinco-ensayos-aufsc3a4tze-und-vortrc3a4ge-1922-1937 husserliana-xxvii-2002.pdf

McNeill, P. (1989). Research Ethics Review in Australia, Europe, and North America. IRB: Ethics \& Human Research, (11)3, 4-7. 
Morales J., Nava G., Esquivel J. y Díaz L., (2011). Principios de ética, bioética y conocimiento del hombre. Universidad Autónoma del Estado de Hidalgo Instituto de Ciencias de la Salud Área Académica de Medicina Área Académica de Farmacia.

Moustakas, C.E. (1994). Phenomenological research methods. Thousand Oaks, CA: Sage.

Mehra, B. (March, 2002). Bias in qualitative research: Voices from an online classroom. The Qualitative Report, (7)1. Recuperado el 10 de diciembre de 2016, en: http://www.nova.edu/ssss/QR/QR71/mehra.html

National Advisory Board on Research Ethics. (2009). Finnish Advisory Board on Research Integrity (TENK). Recuperado el 01 de marzo de 2016, en: http://www.tenk.fi/sites/tenk.fi/files/ethicalprinciples.pdf

Romero C. (2013). La ética en la investigación de las ciencias sociales y humanas. Revista Investigium IRE, Ciencias Sociales y Humanas. V4, (1)

San Martín (2007). Presencia implícita y explícita de temas éticos en investigaciones y ensayos educativos actuales. RMIE, octubre-diciembre 2007, vol. 12, núm. 35, pp. 1289-1308

Thomas, S. y Pollio, H. (2002). Listening to patients: A phenomenological approach to nursing research and practice. New York, NY: Springer.

Van Den Hoonaard, W. (2011). The Seduction of Ethics. Transforming the Social Sciences. Toronto: University of Toronto Press. Recuperado el 29 de febrero de 2016, en: https://books.google.com.mx/books?id=CvjlEQkO2WkC\&pg=PA29\&dq=humanities+researchers+ ethics\&hl=es\&sa=X\&redir_esc=y\#v=onepage \&q=humanities\%20researchers\%20ethics\&f=false

Vanegas, J. H. (2010). Ética y derechos humanos en el marco de la Constitución Política de Colombia de 1991. Revista Jurídicas, 7(1), 74-92. Recuperado el 04 de noviembre de 2015, en: http://dialnet.unirioja.es/servlet/articulo?codigo $=3360752$

Willig, C. y Stainton, W. (2007). The Sage handbook of qualitative research in psychology. Great Britain. SAGE Publication Ltd.

World Medical Association. (2008). Declaración de Helsinki de la Asociación Médica Mundial. Principios éticos para las investigaciones médicas en seres humanos. World Medical Association. Recuperado el 09 de noviembre de 2015, en: http://www.wma.net/es/30publications/10policies/b3/17c_es.pdf

Wynn, L., Israel, M., Thomson, C., White, K. y Carey-White, L. (2014). A national survey of experiences with ethics. The Australian Journal of Antropology, 25(3), 375-377.

Wynn, L. (2016). The Impact of Ethics Review on a Research-Led University Curriculum Results of a Qualitative Study in Australia. Journal of Empirical Research on Human Research Ethics, 11(2), 180190 
Zito, G.I. y Magalháes, B. M. (2015). Research ethics in the dynamic of scientific field: challenges in the building of guidelines for social sciences and humanities. Ciência \& Saúde Coletiva, 20(9), 26152624. 